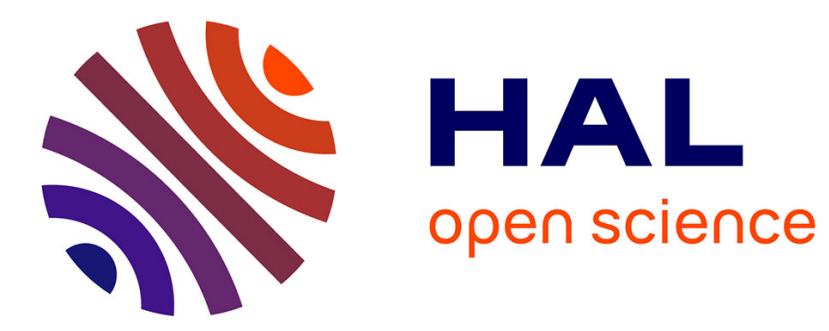

\title{
Digital Holography at Ultimate Shot Noise Level
}

Fadwa Joud, Michael Atlan, Michel Gross

\section{To cite this version:}

Fadwa Joud, Michael Atlan, Michel Gross. Digital Holography at Ultimate Shot Noise Level. Digital Holography and Three-Dimensional Imaging, Apr 2010, Miami, United States. hal-00777474

\section{HAL Id: hal-00777474 https://hal.science/hal-00777474}

Submitted on 18 Jan 2013

HAL is a multi-disciplinary open access archive for the deposit and dissemination of scientific research documents, whether they are published or not. The documents may come from teaching and research institutions in France or abroad, or from public or private research centers.
L'archive ouverte pluridisciplinaire HAL, est destinée au dépôt et à la diffusion de documents scientifiques de niveau recherche, publiés ou non, émanant des établissements d'enseignement et de recherche français ou étrangers, des laboratoires publics ou privés. 


\title{
Citation
}

F. Joud, M. Atlan, and M. Gross, "Digital Holography at Ultimate Shot Noise Level," in Digital Holography and Three-Dimensional Imaging, OSA Technical Digest (CD) (Optical Society of America, 2010), paper DWA2. http://www.opticsinfobase.org/abstract.cfm?URI=DH-2010-DWA2

\section{Digital Holography at Ultimate Shot Noise Level}

\author{
F. Joud ${ }^{1}$, M. Atlan ${ }^{2}$ and M. Gross ${ }^{1}$ \\ ${ }^{1}$ Laboratoire Kastler-Brossel: UMR 8552 CNRS, 'Ecole Normale Supérieure, Université Paris 6; 24 rue Lhomond 75231 Paris Cedex 05 \\ France \\ ${ }^{2}$ Institut Langevin: UMR 7587 CNRS INSERM, ESPCI ParisTech, Universit'e Paris 7, 10 rue Vauquelin, 75231 Paris Cedex 05, \\ gross@lkb.ens.fr
}

\begin{abstract}
We present an Off-Axis Phase-Shifting Digital Holographic technique able to make digital holography at shot noise level. We discuss the advantages of this technique and we give application examples.

OCIS codes: (090.0090) Holography; (0.090.1995) Digital holography, (120.2880) Holographic interferometry
\end{abstract}

\section{Introduction}

Digital holography is a fast-growing research field that has drawn increasing attention. In digital holography, contrarily to classic holography with photographic plate [1], a CCD detector records the holograms, and a computer digitally reconstructs the images [2], avoiding photographic processing. There are two main configurations of Digital Holography: Off-Axis holography [2], which is the oldest and the simplest holographic configuration, and Phase-Shifting holography [3], where several holograms, obtained for different phase of the reference beam or local oscillator beam (LO), are recorded. Heterodyne Holography [4] is a variant of Phase-Shifting holography where acousto-optic modulators provide the LO beam phase shift thus allowing a full control of its amplitude, phase [5] and frequency. Off-Axis Heterodyne Holography has been introduced later in order to perform holography with ultimate sensitivity in the detection of the object signal [6]. We will present here an Off-Axis Phase-Shifting Digital Holography technique that combines the two configurations and thus offer a full control of the Reference Holographic Beam. We will discuss the advantage of this technique, which makes possible to do holography at shot noise level, and we will give application examples.

\section{Setup for the full control of the Reference Holographic Beam}

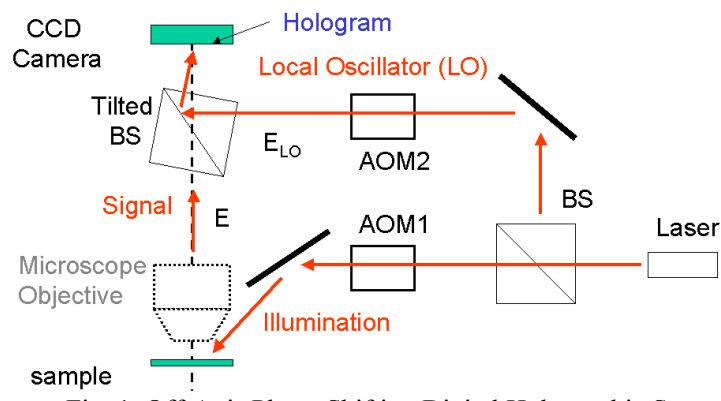

Fig. 1. Off-Axis Phase-Shifting Digital Holographic Setup

The typical Off-Axis Phase-Shifting holographic setup with full control of the Reference Holographic Beam is shown on Fig.1. The setup we have considered here is in reflection geometry. However, transmission geometry can also be considered. The choice of the microscope objective to be used in holographic microscopy is optional. A beam splitter BS splits the laser beam (optical frequnecy $f_{L}$ ) into an illumination beam and a LO beam. The two beams are controlled by two acousto-optic modulators (AOM1 and AOM2), which are driven by sine wave signals of frequencies $f_{1}$ and $f_{2}$ for the illumination and the LO beams respectively (where $f_{1}, f_{2} \cong 80 \mathrm{MHz}$ ). This allows to fully control the phase of the LO beam with respect to the illumination beam. For example, to perform a PhaseShifting with 4 phases detection, at the illumination frequency $f_{L}+f_{1}$, one must choose:

$$
f_{2}-f_{1}=f_{C C D} / 4
$$


where $f_{C C D}$ is the CCD camera frame frequency. Similarly, if one wants to perform 3 phases detection at a frequency shifted by $d f$ with respect to the illumination, one must choose:

$$
f_{2}-f_{1}=\left(f_{C C D} / 3\right)+d f
$$

\section{Shot Noise Holography}
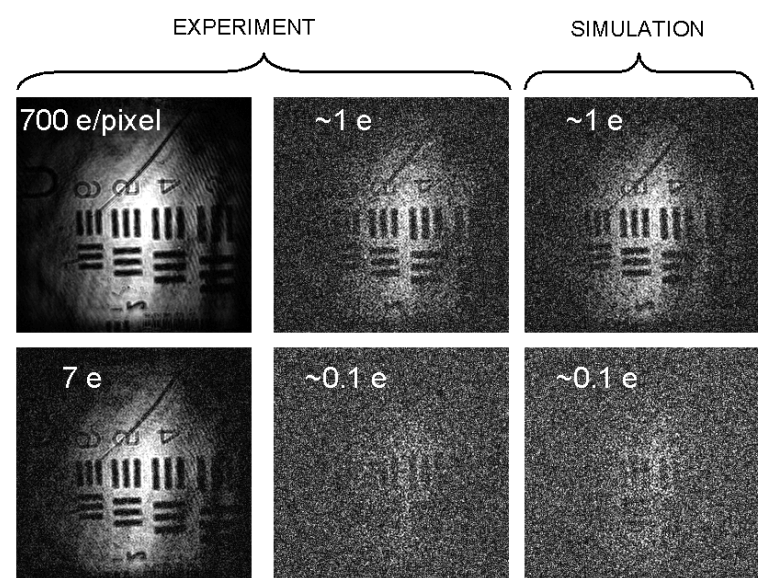

Fig. 2. Reconstructed intensity image $\left(|E|^{2}\right)$ of an USAF target in an off-axis transmission geometry with 4 phases detection on a sequence of 12 frames. Images are obtained with 700, 7, 1, and 0.1 photo electrons (e) per pixel for the 12 frames (i.e 700/12, 7/12 ... for 1 frame). During the reconstruction, spatial filtering is done within the reciprocal space [8]. One must notice that the considered number of pixel to get 700 e per pixel ( or 7, 1...) is the number of pixel within the spatial filter region of interest (200 x 200), and not the size of the reconstruction grid (1024 x 1024).

The holographic setup in Fig.1 offers the capability of performing shot noise holography. Since holography is done in an Off-Axis combined to Phase Shifting mode, the noise of the LO beam can be fully numerically filtered-off [7]. One can thus reach the Ultimate Noise limit [6], which is related to the Poisson Noise of the LO beam photo electronic signal. In a typical situation, the LO signal is about $10^{4}$ e per pixel, and the Poisson noise (about 100 e) is much larger than the reading noise of the camera (30 to 2 e). This means that the camera electronic noise can be neglected, and that the LO beam Poisson noise is dominant. Taking in consideration the holographic (or heterodyne) detection gain, one get an equivalent noise, for the sample signal, of 1 e per pixel for the whole sequence of frames used to image the objet by holography. Figure 2 shows examples of reconstructed images obtained under very weak illumination signal conditions. The simulated images of Fig.2 are obtained by adding the expected amount of Poisson noise to the obtained lowest noise image (700 e per pixel), which is considered here as noiseless.

\section{Control of the holographic detection frequency}
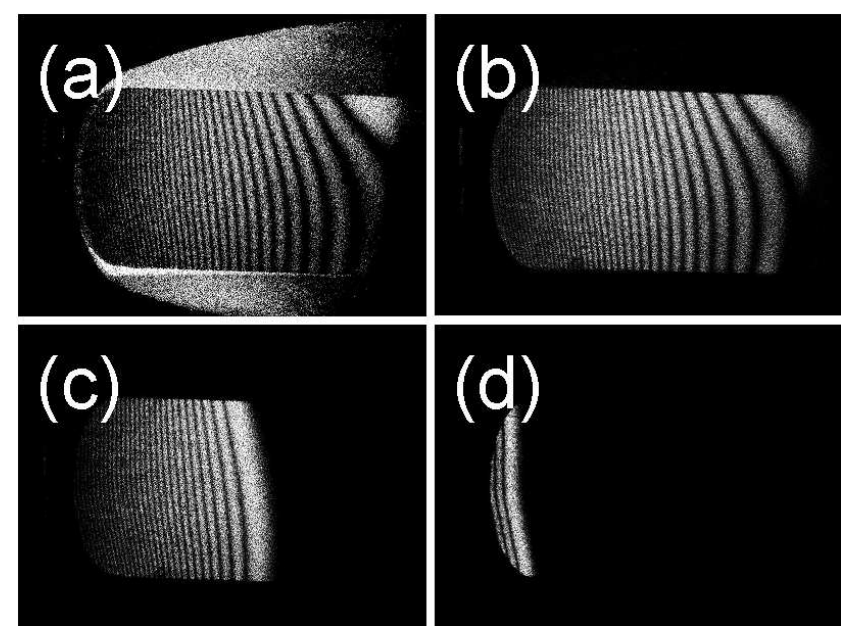

Fig. 3. Reconstructed intensity image $\left(|E|^{2}\right)$ of a vibrating clarinet reed. Image at the illumination frequency (a), at the first sideband frequency $n=1(\mathrm{~b})$, at sideband $\mathrm{n}=20(\mathrm{c})$, at sideband $\mathrm{n}=100(\mathrm{~d})$. 
The holographic setup in Fig.1 is able to control the frequency of the holographic detection. It is then possible to perform the holographic detection at a frequency different than the illumination. If the object vibrates, the phase of the light of the object is modulated, and exhibits sidebands of the optical frequency. By holography, one can thus reconstruct an image of the vibrating object at a sideband frequency. Figure 3 show for example holographic sideband images of a vibrating clarinet reed [9]. It is also possible to consider an object whose motion is not periodic. We can consider, for example, the flow of a fluid that diffuses the light. The frequency spectrum of the light diffused by the fluid is shifted and broadened by the flow motion. By selecting the frequency offset $d f$ of the LO beam, one can select and image by holography different components of the spectrum, and thus different flow velocities components [10]. By this way the blood flow within the micro vessels in a mouse cranium is imaged [11]. Figure 4 show examples of a mouse cranium holographic image for different frequency offset $d f$
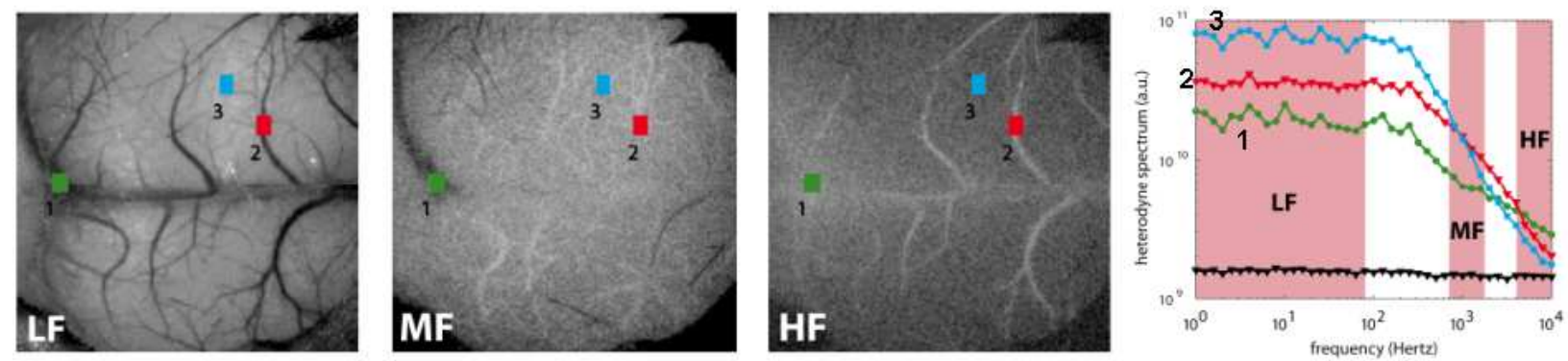

Fig. 4. Reconstructed holographic intensity images of a mouse crania averaged for different frequency offsets df within the intervals $[0,70 \mathrm{~Hz}]$ $(\mathrm{LF}),[0.7,1.6 \mathrm{kHz}](\mathrm{MF})$ and $[4,10 \mathrm{kHz}](\mathrm{HF})$. Average of the reconstructed intensity within the regions of interest 1,2 and 3 as a function of the frequency df.

\section{Conclusion}

By introducing acousto-optic modulators (AOM) on both the illumination and the reference arms within an off-axis geometry of the experimental setup, one can perform digital holography at shot noise level. It is also possible to detect the holographic signal at any frequency close to the illumination beam frequency by properly adjusting the frequencies of the signals that drive the AOMs,. This opens the way to many new applications in the field of digital holography.

[1] D. Gabor. "Microscopy by reconstructed wave-fronts". Proc. Royal Soc. London A, 197, 454-487 (1949).

[2] U. Schnars and W. Juptner. "Direct recording of holograms by a CCD target and numerical reconstruction" Appl. Optics 33, 179-181 (1994).

[3] I. Yamaguchi and T. Zhang. "Phase-shifting digital holography". Optics Letters, 22 : 1268-1270 (1997)

[4] F. Le Clerc, L. Collot and M. Gross. "Numerical heterodyne holography with two-dimensional photodetector arrays" Optics Letters 25 , 716-718 (2000)

[5] M. Atlan, M. Gross and E. Absil. " Accurate phase-shifting digital interferometry" Optics Letters 32, 1456-1458 (2007)

[6] M. Gross and M. Atlan. " Digital holography with ultimate sensitivity" Optics Letters 32, 909-911 (2007)

[7] M. Gross, M. Atlan and E. Absil "Noise and aliases in off-axis and phase-shifting holography" Appl. Optics 47, 1757-1766 (2008)

[8] E. Cuche, P. Marquet and C. Depeursinge "Spatial filtering for zero-order and twin-image elimination in digital off-axis holography" Appl. Opt 39, 4070-4075 (2000)

[9] F. Joud, F. Laloe, M. Atlan and M. Gross “Imaging a vibrating object by Sideband Digital Holography" Opt. Express 17, 2774-2779 (2009)

[10] M. Atlan and M. Gross “Laser Doppler imaging, revisited ” Review of Scientific Instruments, 77, 116103 (2006)

[11] M. Atlan, M. Gross, BC. Forget, T. Vitalis, A. Rancillac, AK.Dunn "Frequency-domain wide-field laser Doppler in vivo imaging" Optics letters, 31, 2762-2764 (2006) 